\title{
Incremental network dysfunction in breast carcinogenesis in constituting progressive DNA Repair attempts within contextual erbb2 Amplification/Overexpression
}

\author{
Lawrence M Agius* \\ Department of Pathology, Mater Dei Hospital, Malta
}

Submission: September 17, 2018; Published: October 09, 2018

*Correspondence Address: Lawrence M Agius, Department of Pathology, Mater Dei Hospital, TAL-QROQQ, University of malta medical school, Msida, Malta, Europe, Tel: 356-21451752; Email: lawrence.agius@um.edu.mt

\begin{abstract}
Performance credentials for the overall network dysfunctions of molecular signatures can be employed as a base-core phenomenon in the classification of breast carcinomas, as well-testified within systems of compound dimension. The incremental dispositions for such appraisal allow a permissive retribution in terms of DNA repair dynamics and as further projected in terms of injury to global genome participation. The realization of DNA injury is further classifiable in terms of an injury that targets defective DNA repair mechanisms and pathways. It is further to such realization that incremental over-expression of the ERBB2 is symptomatic for progression of specific stages characterizing the malignant transformation event.
\end{abstract}

\section{Introduction}

Triple-negative breast neoplasms with negative indices for Estrogen-receptor alpha, ERBB2 and progesterone receptor is a contrasting profile with regard to the often combined positive ERalpha and over-expressed ERBB2 in breast carcinomas. Such dual contrasting profiles indicate the diversity of molecular constitution of breast carcinoma in general and attest to the evolutionary history of a lesion that is based on molecular classifications of the progression of this specific type of neoplasia. The WWOX tumor suppressor gene in epithelial cells modulates gene transcription through interaction with p73, AP-2gamma and ERBB4 proteins; WWOX correlates positively with ER status and genomic instability is driven by loss of WWOX expression [1]. It is further to be realized the dimensions of incremental overexpression of a lacking binding domain for ERBB2 in a manner that is reciprocated in heterodimeric combination with ERBB3 that often activates the PI3K/AKT pathway in inducing cell proliferation of the breast neoplasm. Replication factor $C$ could act as an oncogene or tumor suppressor gene based on the cellular and histologic characteristics of the neoplasm [2].

The conglomerate molecular constitution as positive ERalpha and ERBB2 combination is often susceptible to the action of trastuzumab in dramatically reducing the recurrence of the breast carcinoma lesion. This however is not always observed in terms of negative response to trastuzumab treatment. Nogo-B receptor expression is increased in tamoxifen-resistant breast cancer cells and increases the sensitivity of ERalpha-positive breast cancer to tamoxifen [3]. The complexities of response to molecular amplification or over-expression of ERBB2 is further compounded by non-resolving adjuvant response to trastuzumab administration and may very well constitute a synthetic lethality complex due to the action of two affected gene products. Cues from the breast microbiome and the expression of stressassociated ligands by neoplastic breast duct epithelial cells may shape response to mucosal-associated invariant $\mathrm{T}$ cells during breast carcinogenesis [4].

\section{Complex Heterogeneity}

Complex heterogeneity of combined ERalpha and ERBB2 has emerged in terms of facilitation of the additionally administrated conventional chemotherapeutic agents to the trastuzumab regime. It is in terms of such combined therapy that trastuzumab constitutes a viable option in therapy in terms of either adjuvant agent but also in terms of first line treatment in patients with primary breast carcinoma. Concurrent applied therapeutics in breast neoplasia patients constitutes the dimensional interplay of substantial facilitation and also suppression components of many signaling pathways.GATA4 plays a tumor-suppressive role via repression of NF-kappaB signaling in breast cancer cells [5]. It is further confirmatory evidence that whole networks of the ERBB pathway network constitutes an intricate response paradigm within systems for patients such lesions as mutations of the PI3K and reduced expression of PTEN. These are well-documented in systems of reappraisal and as evidenced in patients with dual 


\section{Cancer Therapy \& Oncology International Journal}

positivity for ERalpha and ERBB2 over-expression. Breast cancer prognosis signature aids in linking risk stratification to disease subtypes; these constitute breast cancer as a very complex and heterogeneous disease with variable molecular mechanism of carcinogenesis and clinical behaviour [6]. TRIM 59 over expression correlates with poor prognosis and contributes to progression of breast carcinoma through AKT signaling pathway [7].

Facilitated sensitivity to conventional chemotherapeutic agent action is well-exemplified in patients with mutations of BRCA1/BRCA2 gene network. It is further to such considerations that the overall dimensions for progression of a breast carcinoma is constitutively reaffirmed in terms of the overall expression of molecular pathway/network dimensions of the ERBB2/ERBB3 combined complex. This is well-evidenced by reduced recurrence of the neoplasm. A novel concept of breast carcinogenesis characterizes malignant transformation as clinical manifestation of underlying metabolic insufficiencies [8].

\section{Molecular Dynamics}

The molecular dynamics of ERBB2/ERBB3 complex have been evidenced by the action of trastuzumab in terms of incremental or decreased over-expression of this molecular complex. The evolutionary consequences of endocrine manipulation in the therapeutic or adjunct response of the breast carcinoma is affirmed consequence to the molecular identity and constitution of the primary breast lesion. This occurs in terms evidenced by insufficiency cooperative dimensions of the growing and spreading neoplasm. RAD51D is involved in DNA double-strand break repair by homologous recombination and is implicated in the maintenance of genomic stability [9].

It is significant to consider the performance of a breast neoplasm as substantial verification of the injury to the cellular DNA. Multiple repair mechanisms are operative in terms of singlestrand breaks, double-strand breaks, inter- and intra-strand crosslinkages and of base-substitution. Further confirmatory lesions relate in particular to the inter-activities of ERalpha positivity with the over-expression of the ERBB2 and also combinatory dimensions of ERBB3. Imbalance of the reciprocally inhibitory loop between the ubiquitin-specific protease USP54 and EGFR/ PI3K/AKT drives breast cancer genesis [10].

\section{Performance Strategies}

Performance strategies incrementally exploit the molecular landscape of breast lesions in terms that further confirm the realization of injury to DNA in carcinomatous cells. Targeted next-generation sequencing identifies clinically relevant somatic mutations in a large cohort of inflammatory breast cancer [11]. System pathway dimensions attest to a molecular complexity that promotes the over-expression of the ERBB2 moiety as reflection of the amplicon at 17q11-24 site in the parent DNA molecule. The outcome dynamics of consequence realize the dimensions of a network operability that includes susceptibility to the poly (ADPribose) polymerase 1 inhibitors that sensitize tumor cells to the action of chemotherapy agents.
System profile convergence is reflected in the overall characterization of DNA injury in terms especially of homologous recombination with the employment of sister DNA strand in guiding the repair of the single strand break. RAD50 germline mutations are associated with poor survival in BRCA1/2negative breast cancer patients and is a highly conserved DNA double-strand break repair gene [12]. Performance confirms the disruption of DNA lesioned sites as evidential reappraisal of an injury that may progress in its own right and further promote such lesions as double-strand breaks. Resveratrol prevents the repair of double strand breaks and sensitises cisplatin-resistant MCF-7 breast cancer cells [13]. PARP1 acts as a sensor for DNA lesions and executes dimensions for reappraisal of progression as welltestified within the system complexity of network functionality and dysfunctionality.

\section{Erbb2 Over-Expression}

Over-expression of ERBB2 may also occur in the absence of the amplicon on chromosome 17. The complex landscape in carcinomatous cells is compounding influence in the context for progression of lesions and of novel lesions within the DNA complement that operates especially in association with defects in the DNA repair mechanisms. Genomic instability as a constitutive hallmark of malignant transformation of the targeted cancer cells is further compounded by a progression that is confirmatory evidence for widespread potential for spread and metastasis. Single nucleotide polymorphisms may modify the risk of cancer and be regarded as potential markers of carcinogenesis; no correlation has been found between single nucleotide polymorphisms of repair genes and tumor grading nor the lymph node status in breast carcinoma [14]. The compound dimensions recur with the systems of progression that underlie the system network realization in metastatic lesions in various organs. Performance retribution is hence inherent in the induced proposed contexts for amplification and over-expression of the ERBB2 relative to the latter's combination with ERBB3.

\section{Conclusion}

Performed progression of the breast carcinoma lesion is appraised inducement for the global context of progression as constituted by defects in the DNA repair mechanisms. The cytoplasmic kinase domain of ERBB2 is constitutive in terms of an amplicon that is dysfunctionally over-expressed. Dysregulated DNA repair and cell proliferation controls are essential driving forces in mammary carcinogenesis [15]. The further emergence of DNA lesions is reflected in mutability of genes such as the 110alpha, 110beta and 110gamma as further attested by network dysfunction. The realization of recurrence of a carcinomatous lesion is projected in terms of constitutive over-expression of ERBB2 as specifically combined with ERBB3.The injury to DNA often promotes error-prone mechanisms of attempted DNA repair as evidenced by system networks incorporating the endocrine micro-environment. The overall systems of cooperative dimension allow for the emergence of DNA lesions that somehow operate as cause/effect in the progression of malignant transformation. 


\section{Cancer Therapy \& Oncology International Journal}

Increments of DNA injury are integrated and inherent dimensions in terms of an amplicon that induces directly the over-expression of the ERBB2.

\section{References}

1. Pospiech K, Pluciennik E, Bednarek AK (2018) WWOX tumor suppressor gene in breast cancer, a historical perspective and future directions. Front Oncol 8: 345.

2. Li Y, Gan S, Ren L, Yuan L, Liu J, et al. (2018) Multifaceted regulation and functions of replication factor $\mathrm{C}$ family in human cancers. Am J of Cancer Research 8(8): 1343-1355.

3. Gao P, Wang X, Jin Y, Hu W, Duan Y, et al. (2018) Nogo-B receptor increases the resistance to tamoxifen in oestrogen receptor-positiver breast cancer cells. Breast Cancer Res 20(1): 112.

4. Zumwalde NA, Haag JD, Gould MN, Gumperz JE (2018) Mucosa associated invariant $\mathrm{T}$ cells from human breast ducts mediate a Th17skewed response to bacterially exposed breast carcinoma cells. Breast Cancer Res 20(1): 111.

5. Han X, Tang J, Chen T, Ren G (2018) Restoration of GATA4 expression impedes breast cancer progression by transcriptional repression of ReLA and inhibition of NF-kB signaling. J Cell Biochem.

6. Yu F, Quan F, Xu J, Zhang Y, Xie Y, et al. (2018) Breast cancer prognosis signature: linking risk stratification to disease subtypes. Brief Bioinform.

7. Liu Y, Dong Y,Zhao L, Su L, Diao K, et al. (2018) TRIM59 over expression correlates with poor prognosis and contributes to breast cancer progression through AKT signaling pathway. Mol Carcinog.
8. da Silva I, da Costa Vieira R, Stella C, Loturco E, Carvalho AL, et al. (2018) Inborn-like errors of metabolism are determinants of breast cancer risk, clinical response and survival: a study of human biochemical individuality. Oncotarget 9(60): 31664-31681.

9. Chen X, Li Y, Ouyang T, Li J, Wang T, Fan Z et al. (2018) Associations between RAD51D gremlin mutations and breast cancer risk and survival in BRCA 1/2-negative breast cancers. Ann Oncol.

10. He L, Liu X, Yang J, Li W, Liu S, et al. (2018) Imbalance of the reciprocally inhibitory loop between the ubiquitin-specific protease USP43 and EGFR/PI3K/AKT drives breast carcinogenesis. Cell Res 28(9): 934951.

11. Liang X, Vacher S, Boulai A, Bernard V, Baulande S, et al. (2018) Targeted next-generation sequencing identifies clinically relevant somatic mutations in a large cohort of inflammatory breast cancer" Breast Cancer Res 2(1): 88

12. Fan C, Zhang J, Ouyang T, Li J, Wang T, et al. (2018) RAD50 gremlin mutations are associated with poor survival in BRCA1/2-negative breast cancer patients. Int J Cancer 143(8):1935-1942.

13. Leon-Galicia I, Diaz-Chavez J, Albino-Sanchez ME, Garcia-Villa E Bermudez-Cruz R, et al. "Resveratrol decreases RAD51 expression and sensitises cisplatin-resistant MCF-7 breast cancer cells. Oncol Rep 39(6): 3025-3033.

14. Smolarz B, Brys M, Forma E, Zadrozny M, Bierkiewicz J, et al. (2017) Data on single nucleotide polymorphism of DNA repair genes and breast cancer risk from Poland. Pathol Oncol Res.

15. Droz-Rosario R, Lu H, Liu J, Liu NA, Gansant S, et al. (2017) Roles of BCCIP deficiency in mammary tumorigenesis. Breast Cancer Res 19(1): 115

\section{Your next submission with Juniper Publishers will reach you the below assets}

- Quality Editorial service

- Swift Peer Review

- Reprints availability

- E-prints Service

- Manuscript Podcast for convenient understanding

- Global attainment for your research

- Manuscript accessibility in different formats ( Pdf, E-pub, Full Text, Audio)

- Unceasing customer service

Track the below URL for one-step submission https://juniperpublishers.com/online-submission.php 